\title{
Development of a Multi-Objective Genetic Algorithm for the Design of Offshore Renewable Energy Systems
}

\author{
Ajit C. Pillai, Philipp R. Thies, and Lars Johanning \\ Renewable Energy Group, \\ College of Engineering, Mathematics, and Physical Sciences, \\ University of Exeter, Penryn, United Kingdom, \\ a.pillai@exeter.ac.uk \\ p.r.thies@exeter.ac.uk \\ 1.johanning@exeter.ac.uk
}

\section{Abstract}

This paper describes the development of a framework using a genetic algorithm in order to aid in the design of a mooring system for offshore renewable energy devices. This framework couples numerical models of the mooring system and structural response to cost models in order for the genetic algorithm to effectively operate considering multiple objectives. The use of this multi-objective optimization approach allows multiple design objectives such as minimum breaking load and the material cost to be minimized simultaneously using an automated mathematical approach. Through the application of this automated approach, a wider set of designs will be considered allowing the system designers to select a design which appropriately balances the trade-off between the competing objectives. In this work, a set of mooring designs that represent efficient solutions for the stipulated constraints are found and presented. The developed framework will be applicable to other offshore technology subsystems allowing multi-objective optimization and reliability to be considered from the design stage in order to improve the design efficiency and aid the industry in using more systematic design approaches.

2. Keywords: mooring system, multi-objective optimization, renewable energy systems, genetic algorithm

\section{Introduction}

As the renewable energy sector continues to grow, it has become increasingly important to offer designs and solutions which are as efficient as possible. With this aim in mind, the field of optimization has increasingly been applied to all engineering disciplines including offshore renewable energy offering both improvements in design and insight into the dynamics of an engineering problem. The present work explores the development of an optimization framework for mooring systems using a multi-objective genetic algorithm (MOGA). This work builds on existing optimization work in the renewable energy field $[1,2]$ making use of a genetic algorithm (GA).

For floating marine renewable energy extraction such as floating wind, wave, and tidal energy installations, the mooring systems is designed to ensure that the structure will stay on station and be able to operate for the duration of the project lifetime, up to 25 years. However, in the design of these systems it is not yet common practice to include an optimization algorithm exploring how changes in the mooring design can impact the various competing objectives that a system designer needs to consider. By introducing a multi-objective optimization strategy to this design problem it is possible to explore a range of design philosophies thereby offering greater clarity on the potential design trade-offs for a decision maker. Furthermore, by employing an optimization approach a greater variety of design alternatives can be explored potentially identifying innovative site-specific mooring systems that would not be considered under standard design approaches.

The optimization routine implemented here seeks to minimize the material cost of the mooring system and the peak tension in the mooring lines. These have been selected to develop the methodology and further objectives can easily be explored in the future depending on the criteria of interest to the mooring system designer and decision makers. To assess the suitability of a given mooring design, the optimization routine makes use of Orcina's OrcaFlex software.

This paper presents the developed methodology and using the Wave Hub site in Cornwall, United Kingdom, a case study is explored demonstrating the capabilities of this approach for the optimization of the mooring system for the OC4 semi-submersible [3] designed for use with floating offshore wind turbines. This study highlights how 
the methodology can aid in decision making and justify engineering design decisions.

\section{Methods}

\subsection{Process Overview}

The industry standard approach for the design of mooring systems for offshore renewable energy systems takes an iterative approach making use of past experience and lessons learned across both the renewable energy sector and the oil and gas sector. Through this approach, feasible mooring systems can be designed and assessed [4]. In general, however, the iterative approach makes use of a deterministic design logic [5], resulting in a limited exploration of the full range of potential designs thereby avoiding more innovative solutions which may meet the requirements.

The developed framework is written in Python 3.5.2 and has been developed in a modular approach allowing different optimization parameters, algorithms, and objectives to be interchanged with a minimal change in the code structure. This has allowed the optimizer to first be developed as a traditional single-objective genetic algorithm (GA) and then transitioned to a MOGA without needing to alter the central structure of the GA or the genome.

As this optimization problem addresses the design of mooring systems, OrcaFlex, an industry standard software package for the analysis of mooring systems has been linked to the optimizer allowing the optimization to use this software package to inform changes in the mooring system.

\subsection{Genetic Algorithm}

GAs represent a family of bio-inspired population based heuristic optimization algorithms that borrow ideas from natural evolution as observed in biological systems. GAs are commonly deployed as they represent a family of generic algorithms which can be applied to a wide range of problems of varying degrees of complexity. The GA makes use of a population of solutions which explore the search space and as a result of the population based approach is proficient in avoiding local solutions. As such, GAs have commonly been applied to complex engineering design problems with good quality solutions often being found. An overview of the canonical genetic algorithm is shown in figure 1. In a GA, solutions with a higher quality, referred to as fitness, are deemed to have a higher probability of being selected to contribute to the generation of new solutions. The selected individuals are then recombined in an attempt to create new, better, solutions in crossover, and then randomly modified to avoid local optima in mutation [6].

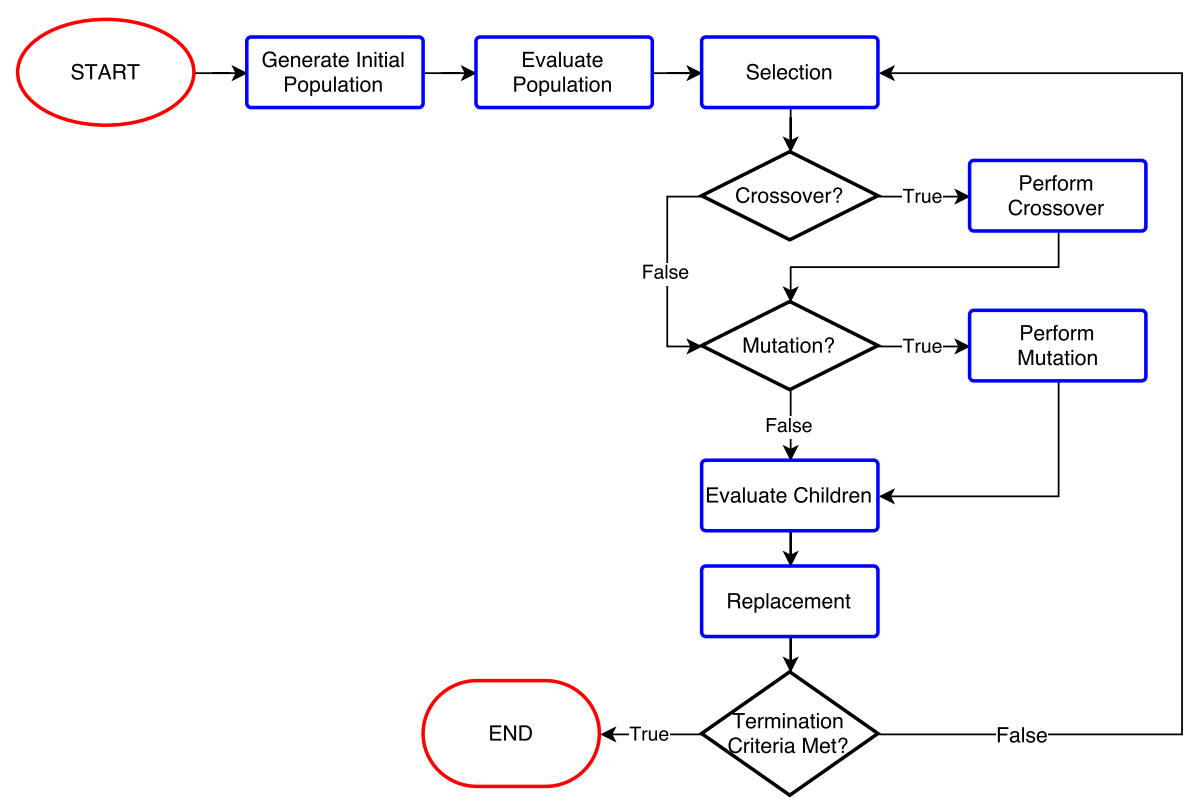

Figure 1: Flowchart of a standard genetic algorithm

In multi-objective optimization, the optimizer seeks to identify a set of solutions which satisfy the trade-off between the competing objectives [7]. Many multi-objective optimization approaches combine the competing objectives in such a way that the problem can be treated as a single objective problem using traditional approaches, however, in 
doing so much of the problem complexity and nuance is lost. True multi-objective optimization is in effect not an extension of single-objective optimization, but requires a different approach all-together in order to simultaneously address the various competing objectives. In a true non-trivial multi-objective optimization problem with conflicting objectives, there is not a single solution which simultaneously optimizes all of the objectives, but a Pareto front which represents the balance between the competing objectives. While an optimization algorithm applied to a single-objective optimization problem seeks to identify a single solution representing the global optima, a multi-objective optimization algorithm seeks instead to identify this Pareto front of potentially an infinite number of solutions.

In traditional single-objective GAs, the fitness of an individual is often used to determine how likely it is to donate genetic material to the next generation, however, in a multi-objective GA, there is no single metric that identifies the quality of a solution, making it more difficult for the algorithm to determine selection. The Non-dominated Sorting Genetic Algorithm-II (NSGA-II) developed by Deb and Pratap [8] is a MOGA which uses a sorting algorithm to identify fronts of non-dominated solutions. Selection occurs based on the relative dominance of solutions and by their crowding distance.

NSGA-II operates by using a sorting algorithm to sort the population on to fronts of non-dominated solutions. These fronts are then ranked and combined with a diversity preservation measure referred to as the crowding distance. The ranked individuals are then used in a traditional tournament selection in which the crowding distance is used as a tie breaker in the event that the two individuals in the tournament are on the same non-dominated front $[7,8]$. From here, standard crossover and mutation operations are used. By using this selection method rather than a traditional selection algorithm, the GA is capable of correctly considering the multiple objectives when determining which individuals should reproduce to create new solutions. The full NSGA-II methodology is well described in Deb and Pratap [8] and Deb [7]. In the present implementation of NSGA-II, the parameters given in table 1 are used as a first step in testing the viability of this approach. It is expected that further tuning of these parameters will be required in further development of this framework in order to improve the quality of the results and avoid premature convergence.

Table 1: Genetic Algorithm Parameters

\begin{tabular}{lr}
\hline Parameter & Value \\
\hline Population Size & 100 \\
Maximum Number of Generations & 50 \\
Crossover Operator & Uniform \\
Mutation Operator & Gaussian \\
Probability of Crossover (Individual) & 0.50 \\
Probability of Crossover (Attribute) & 0.50 \\
Probability of Mutation (Individual) & 0.20 \\
Probability of Mutation (Attribute) & 0.05 \\
\hline
\end{tabular}

\subsection{Mooring Design}

Traditional mooring design for marine renewable energy devices makes use of an iterative design approach in which a starting design is iteratively changed until a set of criteria are met. These criteria are selected to ensure that the designed system can survive the environmental conditions of the selected site for the duration of the project and elicit the desired response for the renewable energy device in question. The criteria therefore often include upper limits for the loads in the lines and at anchors; the maximum excursions for the platform; maximum footprint area of the mooring system; fatigue analysis; and some consideration of the natural frequency of the system [4]. As an iterative approach is taken, the final design is highly dependent on the initial design concept. This initial design is commonly selected based on the experience of the mooring designers and lessons learned across both the oil and gas sector and the renewable energy sector. A number of different starting points may be considered in order to compare the final results, however, the iterative process only ensures that the design criteria are met [5].

The iterative design process of the mooring system can include the consideration of the number of mooring lines, fairlead positions, spreading of mooring lines, size of mooring lines, material of mooring lines, anchor 
design $[4,5,9]$.

4.4 Formulation of Mooring Design Optimization

As all optimization problem seek to minimize or maximize some objective function(s), it is important to define the objectives clearly. In this case, for the design of the mooring system it has been decided to implement a multi-objective approach in which both the peak tension in the lines and the material cost of the mooring lines are minimized.

Building on previous work on the design of mooring systems [10, 11], it was decided to allow the optimizer to select the anchor locations, mooring line lengths, and the mooring line materials. The design of the anchors and the fairlead positions are not design variables in the present optimization problem. The decision variables of the optimization problem are therefore:

- the horizontal distance between the floating body and the anchor [meters];

- the angle between the floating body and the anchor [degrees];

- the length of each section of the mooring line [meters]; and

- the line type for each section of the mooring line

for each line that exists in the OrcaFlex model.

OrcaFlex, developed by Orcina Ltd. is an offshore engineering tool based on finite element analysis that can be used to design and assess mooring configurations. Using the OrcFxAPI provided by Orcina Ltd. [12], the developed Python optimizer is linked to OrcaFlex allowing the mooring configurations proposed by the optimizer to be evaluated using OrcaFlex. Though this is computationally intensive, it has been decided that the additional computational complexity is warranted in order to reduce the uncertainty associated with the assessment of the mooring configurations. The OrcFxAPI Python API allows OrcaFlex models to be accessed, manipulated, and run without launching the OrcaFlex GUI and is therefore well suited to this application. The results of a simulation are also easily accessible through this Python API allowing the optimization routine to both select the model inputs, and process the outputs as required. Figure 2 shows the general flow of the optimizer's evaluation function indicating how the optimizer and OrcaFlex interact through the optimization process. In order to assess the system for the range of wave conditions expected at a site, the wave scatter plot shown in table 2 is used to define the environmental conditions in OrcaFlex.

As the mooring system will respond differently depending on the applied loading condition, it is necessary to evaluate the response and the relevant mooring line tension cycles for each of the wave conditions that occur at the site under consideration. As a result of this, a separate dynamic simulation using OrcaFlex is required for each mooring configuration considered and for each sea state at the site. During each of the dynamic simulations, the optimization constraints are checked in order to ensure that the proposed system is valid. Modelling each of the relevant sea states is also important in order to establish that the mooring line configuration proposed by the optimizer does not exceed the minimum breaking load (MBL) at any point along the mooring lines.

In order to accurately model the design problem it is important to include constraints which limit the search space to feasible solutions. Since the genome contains the line specifications for each line as well as the anchor positions for each line's anchor, the genome is a mixture of various variable types with different ranges. The index values denoted as subscripts of the decision vector for each mooring line $x_{l}$ are therefore specific to the design and implementation of the present genome.

The optimization process is constrained in order to ensure that the solutions proposed by the optimizer represented realistic mooring systems which could potentially be deployed. Therefore, there were limits applied to the anchor position constraining each anchor to be no further than $1000 \mathrm{~m}$ away from the floating platform, and for the mooring lines to stay within a $\pm 15^{\circ}$ range of the original azimuth defined in the OrcaFlex model. The construction of the mooring lines is also limited to the line types predefined in the OrcaFlex model with the optimizer ensuring that any part of the mooring line that is in contact with the seabed is chain. Furthermore, the mooring lines were checked in each simulation to ensure that the MBL is not exceeded. 


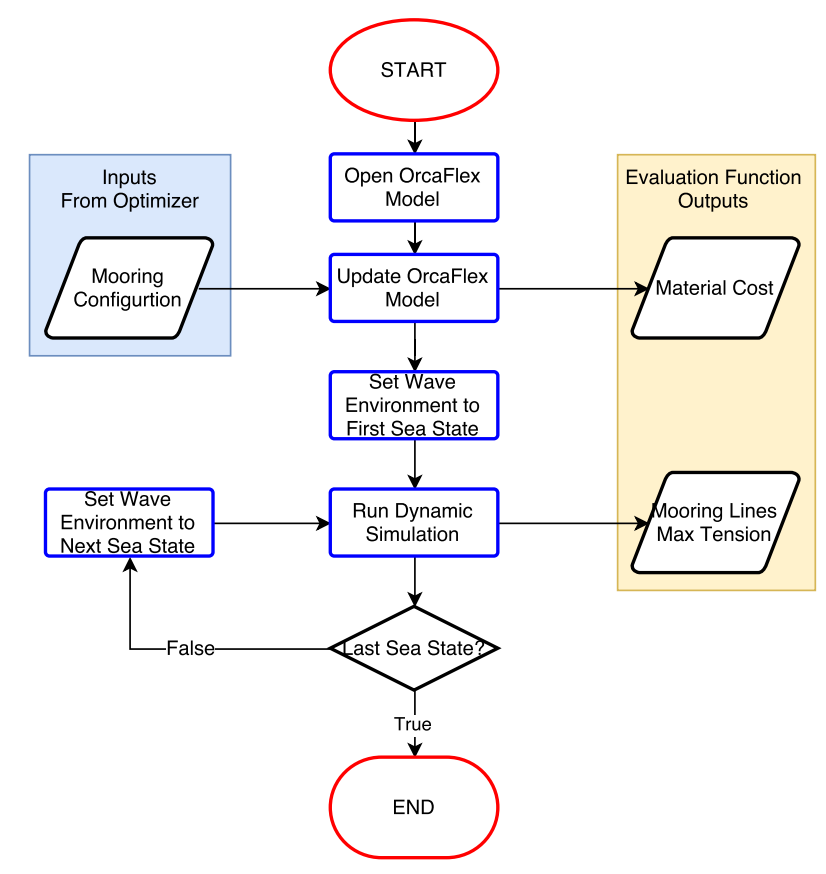

Figure 2: Flowchart of evaluation process for a candidate solution

The formulation of the optimization problem is given in equations 1a-1k. Equation 1a represents the material cost objective while equation 1a defines the minimization of the peak tension objective. Constraints $1 \mathrm{c}$ and $1 \mathrm{~d}$ define the anchor constraints as described above. Constraint $1 \mathrm{f}$ ensures that no point along any of the mooring lines exceeds the minimum breaking load $(M B L)$ of the line type at that given location $a$ along the line. Constraint $1 \mathrm{~g}$ ensures that the line type for each line segment of each mooring line is one of those defined in the OrcaFlex model. Constraint $1 \mathrm{~h}$ ensures that OrcaFlex can successfully calculate the statics for this mooring configuration. Constraint $1 \mathrm{i}$ was introduced to limit the number of line segments, declaring that each mooring line can be constructed out of a maximum of three line segments. Finally constraints $1 \mathrm{j}$ and $1 \mathrm{k}$ identify the line segments of each mooring line that are in contact with the ground during the dynamic simulations and limit these to being chains.

Given the objectives and constraints as described above, the full optimization problem can be formulated as follows:

$$
\begin{array}{lll}
\text { minimize } & f_{1}(x)=\sum_{l \in \mathbb{L}} \sum_{i} c_{i} \cdot x_{l, i} & \forall i \in\left\{2,4,6, \ldots, N_{l}-1\right\} \\
\text { minimize } & f_{2}(x)=\sum_{l \in \mathbb{L}} \max \left(t_{l, a}\right) & \forall s \in \mathbb{S} ; \forall\left\{0<a \leq L_{l}\right\} \\
\text { subject to } & x_{l, 0} \leq 1000 & \forall l \in \mathbb{L} \\
& x_{l, 1} \leq \theta_{l} \pm 15^{\circ} & \forall l \in \mathbb{L} \\
& L_{l}=\sum_{i} x_{l, i}>0 & \forall l \in \mathbb{L} ; \forall i \in\left\{2,4,6, \ldots, N_{l}-1\right\} \\
& t_{l, a} \leq M B L_{a} & \forall l \in \mathbb{L} ; \forall\left\{0<a \leq L_{l}\right\} ; \forall s \in \mathbb{S} \\
& x_{l, i} \in \mathbb{A} & \forall l \in \mathbb{L} ; \forall i \in\left\{3,5,7, \ldots, N_{l}\right\} \\
& V(x)=1 & \\
& \text { count }\left(x_{l, i}\right) \leq 3 & \forall l \in \mathbb{L} ; \forall i \in\left\{3,5,7, \ldots, N_{l}\right\} \\
& G_{l}=\left\{x_{l, i} \mid v_{l, i}=0\right\} & \forall l \in \mathbb{L} ; \forall i \in\left\{3,5,6, \ldots N_{l}\right\} ; \forall s \in \mathbb{S} \\
\text { “chain” } \in G_{l} & \forall l \in \mathbb{L} ; \forall s \in \mathbb{S}
\end{array}
$$

where $f_{1}$ is the first objective function, $f_{2}$ is the second objective function, $f_{3}$ is the third objective function, $x$ represents a candidate solution, $\mathbb{S}$ is the set of sea states that the design must consider, $p_{s}$ is the frequency of occurrence of sea state $s, c_{i}$ is the unit cost of the given segment $i, N_{l}$ is the number of variables associated with line $l, \mathbb{L}$ is the set of mooring lines, $t_{l}$ is the effective tension in line $l$ at the top, $T_{l, p}$ is the tension in line $l$ normalized 
by the MBL, $a$ is a position along the mooring line, $L_{l}$ is the length of mooring line $l, h$ is the water depth, $\theta_{l}$ is the initial orientation of mooring line $l$ relative to the floating body, $\delta_{l}$ is the distance between the floating body and the anchor for mooring line $l, \mathbb{A}$ is the set of available line types, $V(x)$ represents if the static simulation in OrcaFlex can successfully converge for the given candidate solution $x$, and $G_{l}$ is the set of line segments of line $l$ which are in contact with the ground.

Though iterative design approaches may vary the number of mooring lines, it has been decided that for the time being this would be kept constant from the optimizer perspective. The effect of changing the number of mooring lines can still be explored using this framework by running the optimizer successively differing the number of mooring lines input.

\section{Example Case}

\subsection{Case Description}

To explore the applicability of the developed methodology, a case study using the Offshore Code Comparison Collaboration Continuation (OC4) semi-submerisble deployed at Wave Hub has been constructed to explore if the mooring system for the OC4 platform can be altered to better suit the site through use of the present methodology. This platform consists of a main column connected to the turbine tower and three offset columns each of which are connected to the main column. The platform is assumed to have a total of three mooring lines each of which is connected to one of the offset columns. For the purposes of this study, the fairleads are kept the same as in the original definition of the structure [3].

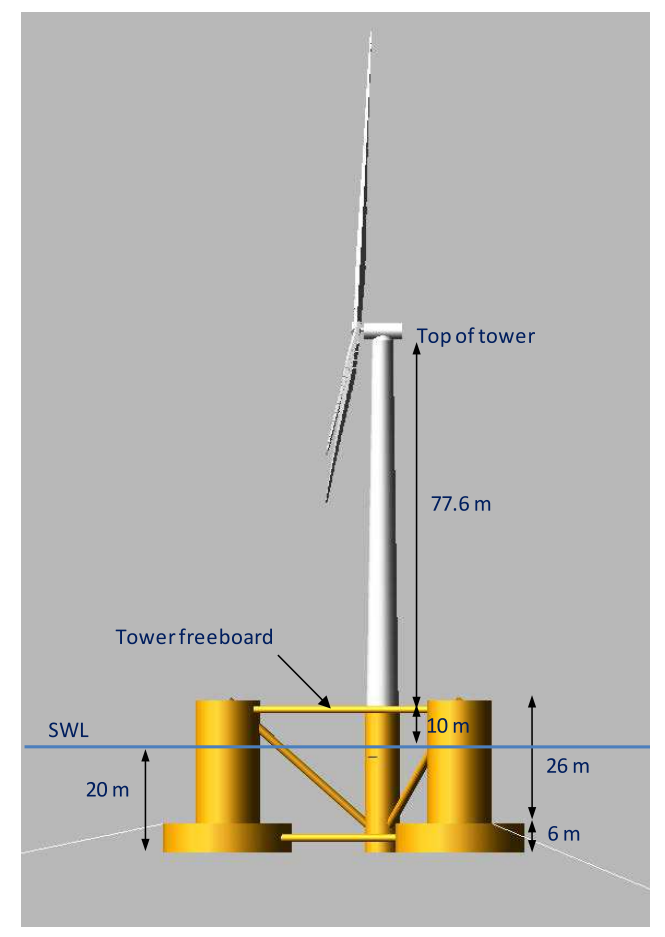

Figure 3: DeepCwind floating wind system used as part of the Offshore Code Comparison Collaboration Continuation (OC4) project [3].

In order to optimize the mooring system for this platform, the full range of sea states described in table 2 are run to evaluate the suitability of the mooring system. A portion of the DTOcean Database [14] was provided to the optimizer allowing it to select from ten line types, six chains and four polyester ropes between $24 \mathrm{~mm}$ and $200 \mathrm{~mm}$ diameters, for each line segment of each mooring line. This component database includes not only the size, MBL, and stiffness of each possible line type, but also the unit material cost of each component.

\subsection{Case Results}

Through the application of this optimization framework, a range of solutions known as the Pareto front are iden- 
Table 2: Wave scatter table for Wave Hub site [13]

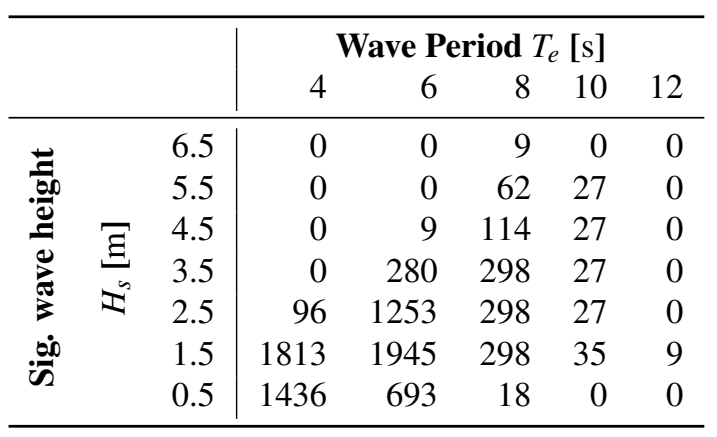

tified. With respect to the two objective functions this front is shown in figure 4. From these results it can be observed that the two objectives are indeed competing and that by minimizing the peak tensions in the mooring system, the material cost of the mooring system increases. Likewise, as one tries to minimize the cost of the mooring system tending towards a taut system, the tension in this mooring system is found to increase.

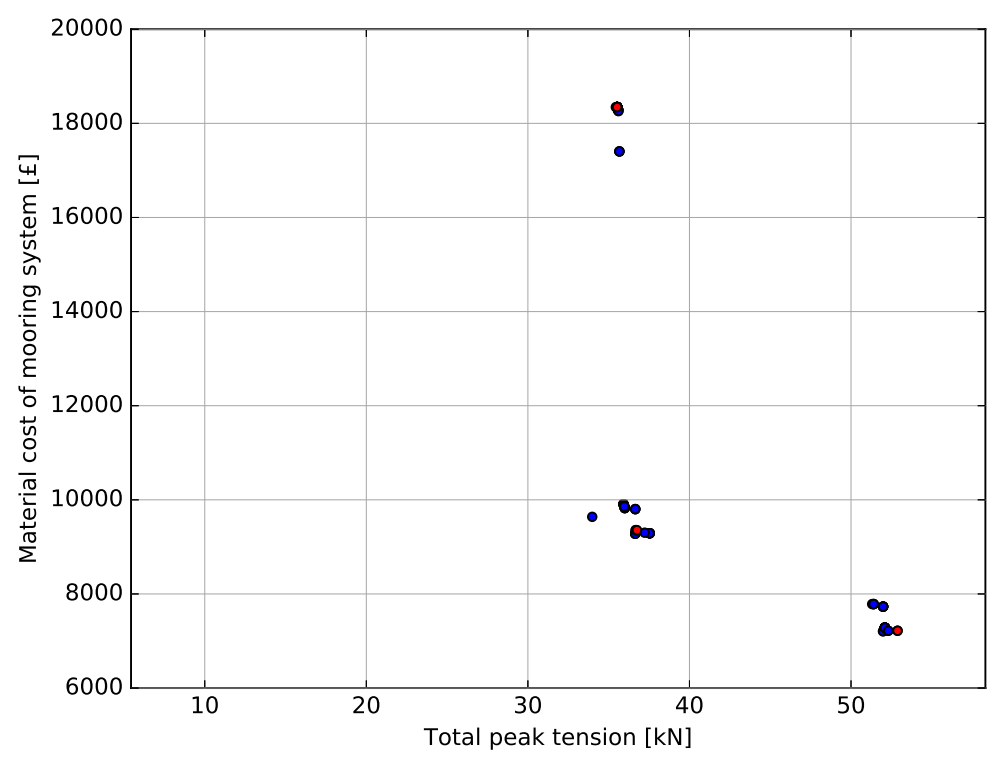

Figure 4: Optimization solutions shown relative to the objective functions. The red points represent solutions described in tables 3-5. The red point at the knee of the curve corresponds to the solution pictured in figure 5 and described in table 3.

The extreme ends of the Pareto front in figure 4 represent the solutions to the respective single objective optimization problems (i.e. either minimizing the material cost of the mooring system or minimizing the peak tensions). The intermediate positions present the compromise between these two extremes allowing the system designer to select designs based on how they value the different constraints.

As can be seen in figure 4, the application of this methodology to the design of the mooring system produces a set of designs ranging between $£ 7,218$ and $£ 18,345$ with respect to the cost objective and $33 \mathrm{kN}$ to $53 \mathrm{kN}$ with respect to the peak tension. The relatively low crossover rate used leads to many repeated individuals in the final population indicated be the high density clusters of solutions along the Pareto front. This highlights the need for further tuning of the GA parameters in order to better define the full range of solutions along the Pareto front. Having said that, the final population does still take on the expected shape identifying the trade-off between the material cost of the mooring system and the total peak tension. The proposed mooring systems range in construction from chain 
only solutions to a combination of chain-polyester or chain-polyester-chain line constructions. The ability for the optimizer to simultaneously select the length of the sections, their construction, and their respective diameters allows the stiffness characteristics of the individual mooring lines to be tuned to the site conditions allowing these objectives to be effectively minimized.

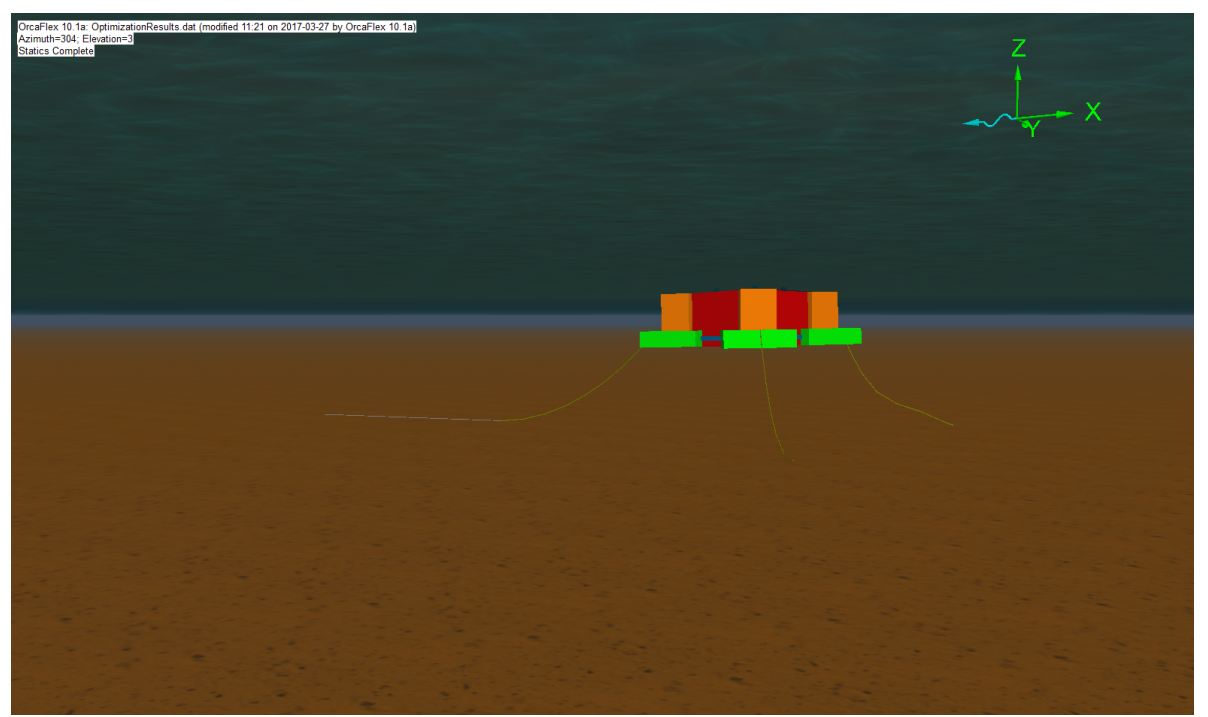

Figure 5: Suggested mooring solution for an efficient solution, balancing cost and mooring peak tensions for the OC4 platform at the Wave Hub site. Mooring solution corresponds to the red point in figure 4 and is described in table 3.

From the Pareto front, the knee of the Pareto curve represents the solutions which most equally balance the competing objectives. The solution given by table 3 and pictured in figure 5 is one of these solutions located at the knee of the Pareto front with objective values of $36 \mathrm{kN}$ and $£ 9,355$. This solution is potentially of interest as very short catenary mooring lines are selected and the mooring system is asymmetrical. Mooring line 1 uses a chainpolyester-chain construction while lines 2 and 3 are both chain. The lengths of all three lines also differ from one another. Mooring line 2 which is aligned with the wave direction makes use of significantly longer segments than lines 1 and 3 and uses only chain construction. This is not entirely unsurprising as because line 2 is aligned with the wave direction it would be expected to experience the highest tensions and a long chain catenary is one effective strategy for mitigating against this. Furthermore, though the anchors were permitted to be up to $1.000 \mathrm{~m}$ away, the present solution is relatively compact with the farthest anchor located only $153 \mathrm{~m}$ away from the platform.

Table 3: Numerical result - knee of Pareto front

\begin{tabular}{rrrrr}
\hline Line & Anchor distance $[\mathrm{m}]$ & Anchor direction $\left[^{\circ}\right]$ & Line length $[\mathrm{m}]$ & Line type \\
\hline 1 & & & 39.29 & 32 mm chain \\
1 & 47.69 & 225.64 & 21.30 & 152 mm polyester \\
1 & & & 4.05 & 32 mm chain \\
2 & 153.10 & 14.03 & 165.00 & 24 mm chain \\
3 & 37.27 & 131.24 & 57.85 & 24 mm chain \\
\hline
\end{tabular}

Examining the extreme values of the Pareto gives the designs defined in tables 4 and 5. These designs correspond to the two extreme red points shown in figure 4 . In table 4 the solution proposed further reduces the peak tensions to $35 \mathrm{kN}$ compared to the knee point, but at the same time significantly increases the cost to $£ 18,345$ as a result of the longer catenary lines used in which the anchors range between $153 \mathrm{~m}$ to $353 \mathrm{~m}$ away from the platform. Alternatively, the mooring system proposed in table 5 has much shorter lines with all anchors less than $48 \mathrm{~m}$ from 
the platform. This design leads to reduced cost relative to both the design at the knee of the Pareto front and the long catenary solution with the total cost only $£ 7,218$. However, as a result of the short lines, the peak tension is increased relative to the other designs to $53 \mathrm{kN}$.

Table 4: Numerical result - cost extreme of Pareto front

\begin{tabular}{rrrrr}
\hline Line & Anchor distance $[\mathrm{m}]$ & Anchor direction $\left[^{\circ}\right]$ & Line length $[\mathrm{m}]$ & Line type \\
\hline 1 & 178.57 & 251.69 & 193.43 & $24 \mathrm{~mm}$ chain \\
2 & 153.10 & 14.03 & 165.00 & 24 mm chain \\
3 & 353.40 & 105.48 & 327.85 & 24 mm chain \\
3 & & & 47.15 & $32 \mathrm{~mm}$ chain \\
\hline
\end{tabular}

Table 5: Numerical result - tension extreme of Pareto front

\begin{tabular}{rrrrr}
\hline Line & Anchor distance $[\mathrm{m}]$ & Anchor direction $\left[{ }^{\circ}\right]$ & Line length $[\mathrm{m}]$ & Line type \\
\hline 1 & & & 39.29 & 32 mm chain \\
1 & 47.69 & 225.64 & 21.30 & 152 mm polyester \\
1 & & & 4.05 & 24 mm chain \\
2 & 15.31 & 356.19 & 43.45 & $32 \mathrm{~mm}$ chain \\
3 & 37.85 & 131.24 & 58.29 & 24 mm chain \\
\hline
\end{tabular}

\section{Concluding Remarks}

The development of a framework for the multi-objective optimization of a mooring system for renewable energy applications has been presented. By considering the range of wave conditions at the deployment site, the application of this methodology allows a site and device specific mooring system to be designed while considering various competing objectives highlighting the trade-offs between these objectives. Through exploration of the solutions highlighted by this methodology the system designers and site developers can better identify mooring system design philosophies which are of interest and can potentially aid in the cost reduction sought by offshore renewable energy developers.

Ongoing work by the authors is exploring further parameter tuning of the genetic algorithm in order to better define the Pareto front as well as the extension of the methodology to include a third objective to consider the impact of fatigue damage on the mooring system. This will allow the mooring system reliability over its lifetime to be explicitly included in the design phase of the mooring system.

\section{Acknowledgements}

This work is funded by the EPSRC (UK) grant for the SuperGen United Kingdom Centre for Marine Energy Research (UKCMER) [grant number: EP/P008682/1].

\section{References}

[1] R. Baños, F. Manzano-Agugliaro, F. Montoya, C. Gil, a. Alcayde, and J. Gómez, "Optimization methods applied to renewable and sustainable energy: A review," Renewable and Sustainable Energy Reviews, vol. 15, no. 4, pp. 1753-1766, may 2011. [Online]. Available: http://linkinghub.elsevier.com/retrieve/pii/S1364032110004430

[2] A. C. Pillai, J. Chick, L. Johanning, M. Khorasanchi, and S. Barbouchi, "Comparison of Offshore Wind Farm Layout Optimizaiton Using a Genetic Algorithm and a Particle Swarm Optimizer," in Proceedings of the ASME 2016 35th International Conference on Ocean, Offshore and Arctic Engineering (OMAE 2016) Busan, South Korea, vol. 6. ASME, 2016, pp. 1-11. 
[3] A. Robertson, J. Jonkman, and M. Masciola, "Definition of the Semisubmersible Floating System for Phase II of OC4," Golden, CO, no. September, p. 38, 2014. [Online]. Available: http://www.nrel.gov/docs/fy14osti/60601.pdf

[4] L. Johanning, G. Smith, and J. Wolfram, "Mooring design approach for wave energy converters," Proceedings of the Institution of Mechanical Engineers, Part M: Journal of Engineering for the Maritime Environment, vol. 220, no. 4, pp. 159-174, 2006. [Online]. Available: http://dx.doi.org/10.1243/14750902JEME54

[5] S. Weller, L. Johanning, L. Victor, J. Heath, J. Eddy, R. Jensen, and S. Banfield, "DTOcean Deliverable 4.5 Mooring and foundation module framework for DTOcean Tool," 2015.

[6] J. H. Holland, Adaptation In Natural And Artificial Systems. [Electronic Resource] : An Introductory Analysis With Applications To Biology, Control, And Artificial Intelligence, 2nd ed. Cambridge, Mass.: MIT Press, 1992.

[7] K. Deb, Multi-Objective Optimization using Evolutionary Algorithms. Chichester: Wiley \& Sons, 2001.

[8] K. Deb and A. Pratap, "A fast and elitist multiobjective genetic algorithm: NSGA-II," IEEE Transactions on Evolutionary Computation, vol. 6, no. 2, pp. 182-197, 2002. [Online]. Available: http://ieeexplore.ieee.org/xpls/abs_all.jsp?arnumber=996017

[9] R. Harris, L. Johanning, and J. Wolfram, "Mooring systems for wave energy converters: A review of design issues and choices," Marec2004, 2004. [Online]. Available: http://abs5.me.washington.edu/pub/tidal_wave/mooringsystems.pdf

[10] M. Shafieefar and A. Rezvani, "Mooring optimization of floating platforms using a genetic algorithm," Ocean Engineering, vol. 34, no. 10, pp. 1413-1421, 2007.

[11] A. J. J. Carbono, I. F. M. Menezes, and L. F. Martha, "Mooring Pattern Optimization using Genetic Algorithms," Structural and Multidisciplinary Optimization, no. June, pp. 1-9, 2005.

[12] D. Heffernan, “An introduction to the Python interface to OrcaFlex," Orcina, Tech. Rep. April, 2016.

[13] E. Pitt, A. Saulter, and H. Smith, "The wave power climate at the Wave Hub site," Applied Wave Research report to SWRDA, Tech. Rep. November, 2006.

[14] JRC Ocean, "DT Ocean Suite - DTOcean Database 1.0.0," 2016. [Online]. Available: https://setis.ec.europa.eu/dt-ocean/download/file/fid/82 